\title{
Protoporphyrin IX-mediated sonodynamic therapy promotes autophagy in vascular smooth muscle cells
}

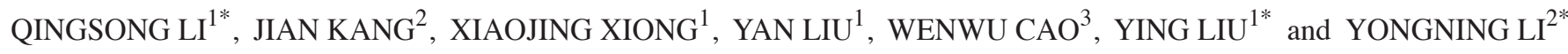 \\ Departments of ${ }^{1}$ Cardiology and ${ }^{2}$ Emergency, The First Affiliated Hospital, Dalian Medical University, Dalian, \\ Liaoning 116011, P.R. China; ${ }^{3}$ Materials Research Institute, Pennsylvania State University, University Park, PA 16802, USA
}

Received July 2, 2016; Accepted April 13, 2017

DOI: $10.3892 / \mathrm{ol} .2017 .6394$

\begin{abstract}
Sonodynamic therapy (SDT) is effective in treating intimal hyperplasia and promoting plaque stability in animal models. The present study aimed to evaluate the effects of SDT with the sonosensitizer protoporphyrin IX (PpIX) on vascular smooth muscle cell (VSMC) viability and autophagy. Cultured VSMCs cells were divided into the following groups: i) Control, ii) ultrasound, iii) PpIX and iv) SDT. Flow cytometry and laser confocal detection were used to measure Annexin V stained VSMCs following different treatments. Alterations in mitochondrial membrane potential (MMP) were evaluated via JC-1 staining. Autophagosome formation was observed using electron and fluorescence microscopy. Western blotting was used to analyze the expression levels of the autophagy markers light chain 3 (LC3-I) and LC3-II. The results demonstrated that SDT did not trigger apoptosis nor induce a significant decline in MMP of VSMCs. However, SDT significantly increased autophagasome formation and increased the LC3-II/LC3-I ratio. The findings demonstrated that PpIX-SDT increased autophagy without inducing mitochondrial-dependent apoptosis in VSMCs.
\end{abstract}

\section{Introduction}

Atherosclerosis is a major cardiovascular disease. Sonodynamic therapy (SDT), which is a noninvasive technique and uses ultrasonic energy to activate certain drugs (i.e. sonosensitizer)

Correspondence to: Dr Yongning Li, Department of Emergency, The First Affiliated Hospital of Dalian Medical University, Dalian, Liaoning 116011, P.R. China

E-mail: lyn1009@126.com

Dr Ying Liu, Department of Cardiologyy, The First Affiliated Hospital of Dalian Medical University, Dalian, Liaoning 116011, P.R. China

E-mail: 18098875801@163.com

*Contributed equally

Key words: sonodynamic therapy, protoporphyrin IX, vascular smooth muscle cells, autophagy, apoptosis to produce chemical reactions and reactive oxygen species, has been shown to have potential to attenuate progression of atherosclerotic plaque formation (1-5).

Currently, porphyrins and their analogs are the predominant sonosensitizers used in the clinic (6). Among these porphyrin-related sonosensitizers, protoporphyrin IX (PpIX) was found to be accumulated specifically in atherosclerotic plaques at a much higher concentration, i.e. 10 times higher, than in normal vessel walls (7), indicating that PpIX is a potential atherosclerosis-selective sonosensitizer.

Cell autophagy, the degradation process of cellular proteins or organelles in the cell (8), is an important defense and protection mechanism. Previous studies have shown that autophagy plays an important role in atherosclerosis $(9,10)$. For instance, autophagy can selectively remove macrophages in the artery atheromatous plaque and participate in degradation of low-density lipoprotein (LDL) cholesterol (11). In addition, induced autophagy in vascular smooth muscle cells (VSMCs) can prevent oxidized LDL (oxLDL)-triggered VSMC foam cell formation (12). In contrast, deficient VSMC autophagy affects blood vessel contractability by changing the $\mathrm{Ca}^{2+}$ steady-state response (13). Therefore, VSMC differentiation and autophagy play important roles in the initiation and development of atherosclerosis, and have been therapeutic targets for treatment of atherosclerosis.

SDT has been shown to promote macrophage apoptosis and maintain stability of atherosclerotic plaques (4); however, the role of VSMCs in the SDT-treated plaques remains unclear. In the present study, we aimed to investigate the PpIX-mediated sonodynamic effects on VSMCs in order to provide molecular mechanisms by which SDT was clinically employed to treat atherosclerosis.

\section{Materials and methods}

Reagents. PpIX was purchased from Sigma-Aldrich, reconstituted in $100 \%$ DMSO (Sigma-Aldrich, St. Louis, MO, USA) to $0.1 \mathrm{~g} / \mathrm{ml}$ and stored at room temperature. The VSMC line A7r5 (ATCC) was derived from murine thoracic vascular aorta. JC-1 probe was provided by Beyotime Institute of Biotechnology (Haimen, China). DAPI and propidium iodide (PI) were obtained from Sigma-Aldrich. Light chain 3B (LC3B) antibody and anti-rabbit secondary antibodies were obtained from Cell Signaling Technology (Danvers, MA, USA). Antibodies 
against microtubule-associated protein 1 LC3 were purchased from Sigma Chemical Co. Fetal bovine serum (FBS) and Dulbecco's modified Eagle's medium (DMEM) were obtained from Hyclone Laboratories, Inc. (HyClone, Logan, UT, USA). All other reagents were obtained from Sigma Chemical Co. Ltd.

Cell preparation and SDT procedure. A7r5 cell line was maintained in DMEM supplemented with $10 \%$ FBS, penicillin, and streptomycin. Cells were grown at $37^{\circ} \mathrm{C}$ in a humidified $5 \%$ $\mathrm{CO}_{2} / 95 \%$ air atmosphere. During the SDT treatment procedure, VSMCs were exposed to ultrasound generated by the ultrasonic generator and power amplifier (Harbin Institute of Technology, Harbin, China) at $1.0 \mathrm{MHz}$ with an intensity of $1.0 \mathrm{~W} / \mathrm{cm}^{2}$ in the presence or absence of PpIX, which was maintained at a concentration of $2 \mu \mathrm{g} / \mathrm{ml}$. During the sonication procedure, the temperature of the solution inside the Petri dishes increased less than $0.5^{\circ} \mathrm{C}$, as monitored with a thermometer.

Flow cytometry analysis. Cells were seeded into $35 \mathrm{~mm}$ Petri dishes and divided into 4 groups: Control group, ultrasound group, PpIX group, and SDT group. Each group consisted of 8 dishes. Five min after ultrasound treatment, cells were placed in a $37^{\circ} \mathrm{C}$ and $5 \% \mathrm{CO}_{2}$ incubator for $6 \mathrm{~h}$, followed by sequential incubation with $5 \mu \mathrm{l}$ Annexin V-FITC for $15 \mathrm{~min}$ and $10 \mu \mathrm{l} \mathrm{PI}$ for $5 \mathrm{~min}$ in the dark room. Apoptosis rates were analyzed by flow cytometry (Epics Altra II; Beckman Coulter, Brea, CA, USA). Experiments were repeated three times for each group under the same conditions.

Laser scanning confocal microscopy. Six h after SDT, cells were incubated with $5 \mu \mathrm{l}$ Annexin $\mathrm{V}$ in the dark for $15 \mathrm{~min}$. Laser scanning confocal microscopy was used to observe apoptosis with excitation at $488 \mathrm{~nm}$ and emission at $525 \mathrm{~nm}$ wavelengths (SP8; Leica, Mannheim, Germany). Experiments were repeated three times for each group under the same conditions.

Mitochondrial membrane potential (MMP) assessment. MMP was assessed using the probe JC-1, a sensitive fluorescent dye used to detect changes in MMP (1). Briefly, $6 \mathrm{~h}$ after SDT, VSMCs were incubated with $10 \mathrm{mg} / \mathrm{ml} \mathrm{JC}-1$ for $10 \mathrm{~min}$ at $37^{\circ} \mathrm{C}$ in the dark and monitored with a fluorescence microscope. Red-orange fluorescence was attributable to a potential-dependent aggregation in the mitochondria. Green fluorescence, reflecting the monomeric form of JC-1, appeared in the cytosol after mitochondrial membrane depolarization.

Transition electron microscopy (TEM). Four groups of cells were collected by centrifugation, washed with $1 \mathrm{X}$ phosphate buffered saline (PBS) (0.01 M pH 7.2-7.4), embedded with agar, fixed with $2.5 \%$ glutaraldehyde, post-fixed with osmium tetroxide, dehydrated in gradient alcohol, and embedded in Epon812 (Yiwei Info Technology Co. Ltd., Shanghai, China). The blocks were then cut into ultra-thin sections, stained with uranium acetate, and observed and photographed under a TEM (Hitachi, Tokyo, Japan).

Immunofluorescence staining and fluorescence microscopy imaging. Six hours after different treatments, VSMCs were washed with $1 \mathrm{X}$ PBS and fixed with $4 \%$ paraformaldehyde for $30 \mathrm{~min}$. VSMCs were then incubated with the primary antibody of interest at $4^{\circ} \mathrm{C}$ overnight. VSMCs were then washed with 1x PBS three times and stained with fluorescent second antibody at $37^{\circ} \mathrm{C}$ for $30 \mathrm{~min}$ in the dark. DAPI was used for nuclear staining. After fluorescence quenching, images were acquired with the same exposure settings using a fluorescence microscopy with standard excitation filters (Olympus Corporation, Tokyo, Japan).

Western blot analysis. SDS-PAGE and immunoblotting were performed according to standard procedures to detect the expression levels of autophagy marker proteins, such as LC3. Briefly, cells were lysed with RIPA buffer on ice, and supernatant samples were resolved in 10-15\% SDS-PAGE depending on the size of the target protein, followed by transfer onto nitrocellulose membranes (Millipore, Billerica, MA, USA). Thereafter, membranes were incubated in blocking buffer at room temperature for $1 \mathrm{~h}$, followed by incubation with anti-LC3 antibody at $4^{\circ} \mathrm{C}$ overnight. The protein-antibody complexes were then tagged with IR Dye 680-labeled secondary antibodies at room temperature for $1 \mathrm{~h}$. The infrared fluorescence was detected with the Odyssey infrared imaging system (Li-Cor Bioscience, Lincoln, NE, USA). $\beta$-actin was used as a loading control. The ratios of LC3-II/LC3-I to $\beta$-actin in each experiment were calculated by Quantity One software (Bio-Rad Laboratories, Inc., Hercules, CA, USA).

Statistical analysis. All values are expressed as means \pm SD of at least three independent experiments, and one-way ANOVA was used to analyze the statistical difference among the multiple groups. A P-value less than 0.05 was considered statistically significant.

\section{Results}

PpIX-SDT did not increase VSMC apoptosis. First, we investigated whether PpIX-SDT had any effect on VSMC apoptosis. VSMCs in SDT and control groups were stained with Annexin V-FITC, followed by flow cytometric analysis. As shown in Fig. 1A, no significant difference in apoptosis between SDT and control group was observed, which was further confirmed by confocal microscopy (Fig. 1B).

PpIX-SDT did not induce loss of MMP in VSMCs. As shown in Fig. 2, normal VSMCs with JC-1 staining exhibited orange fluorescence, and neither light nor PpIX alone induced any changes in MMP. However, SDT treatment induced a diffuse orange staining pattern in VSMCs, which was representative of no significant decline in MMP.

PpIX-SDT induces VSMC autophagy. Next, we explored VSMC autophagic activity in the SDT and other groups with electron microscopy. The number of autophagy bodies, including nuclear pyknosis, karyorrhexis, cell shrinkage, without the formation of apoptotic body, in the SDT group were significantly higher compared to the other groups (Fig. 3). To further confirm that SDT induced autophagy in VSMCs, we measured the levels of LC3-II, a widely used indicator 

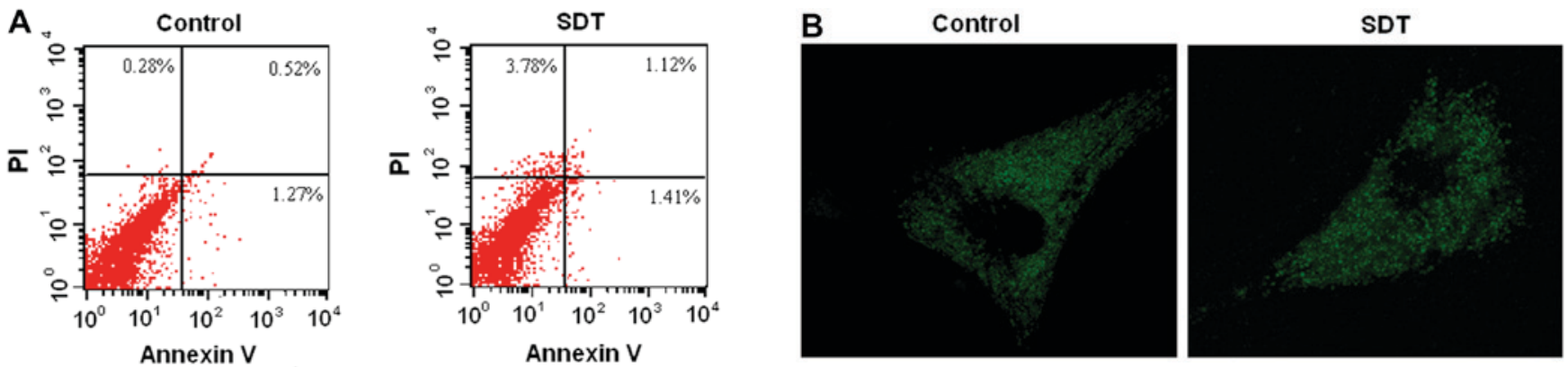

Figure 1. PpIX-SDT does not significantly induce VSMC apoptosis. (A) Flow cytometry with double staining of Annexin V and PI. (B) Confocal microscopy observation with Annexin V low dye in the unimpaired cell membranes.
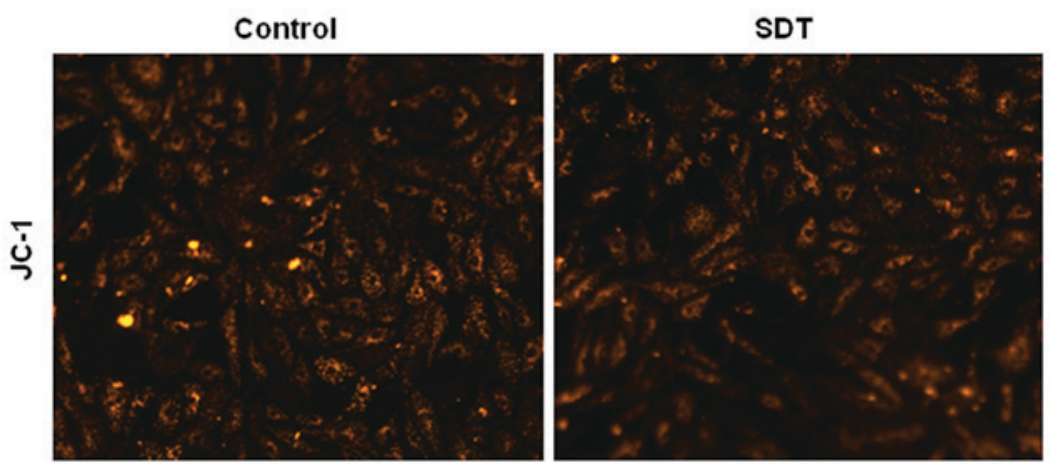

Figure 2. PpIX-SDT does not significantly alter VSMC mitochondrial membrane potential (MMP). Fluorescent probe JC-1 was used to detect changes in MMP. Normal cultured VSMCs contained orange fluorescent mitochondria in the cytoplasm.
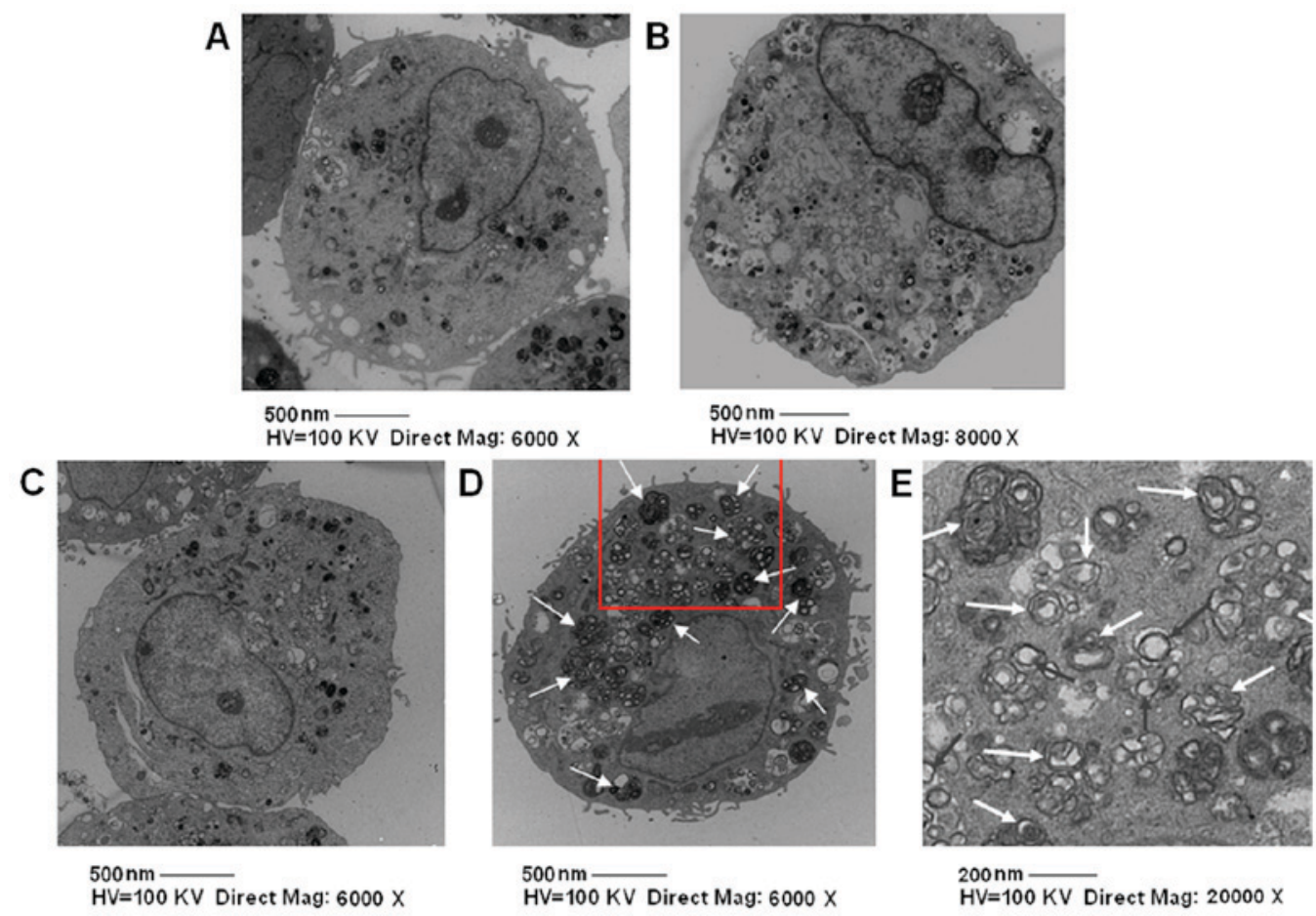

Figure 3. SDT increases autophagosome formation and promotes extensive vacuolization. TEM reveals ultrastructural changes in VSMCs. (A) Control group; (B) PpIX group; (C) ultrasound group; (D) SDT group; (E) autophagosomes (white arrows) were found in the SDT group (magnification, x20,000) and were swollen and vacuolated (black arrows).

of autophagy (13), in VSMCs of different groups. As shown in Fig. 4, a significant increase in fluorescence was observed in the SDT treatment group compared to the control group. We also measured the expression levels of LC3-I and LC3-II in VSMCs in different groups by western blot analysis. As shown in Fig. 5, the LC3-II/LC3-I ratio was significantly increased 


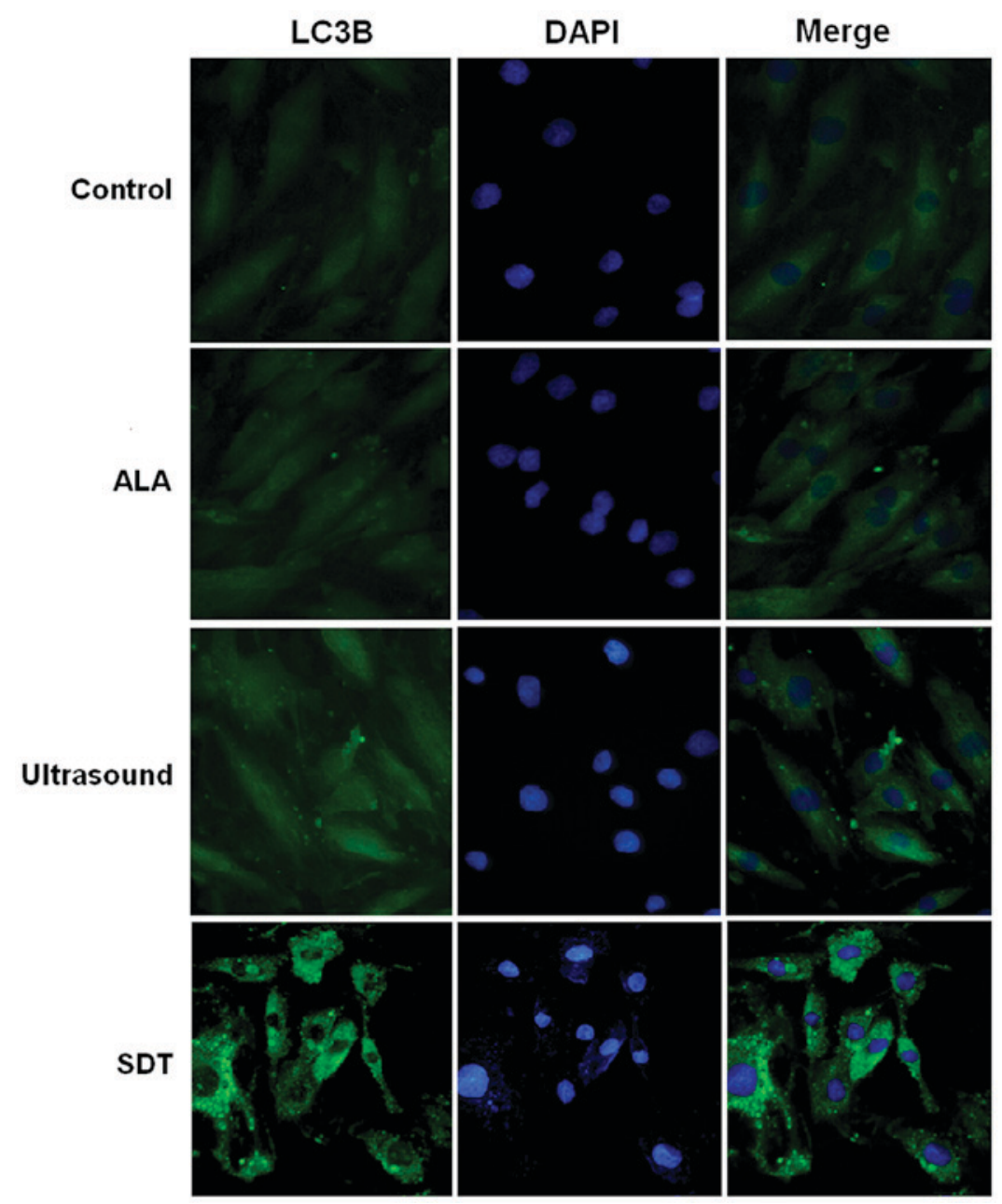

Figure 4. PpXT-SDT increases VSMC autophagic activity. Fluorescence microscopy showed a punctate pattern of fluorescence that was indicative of increased autophagy in VSMCs.
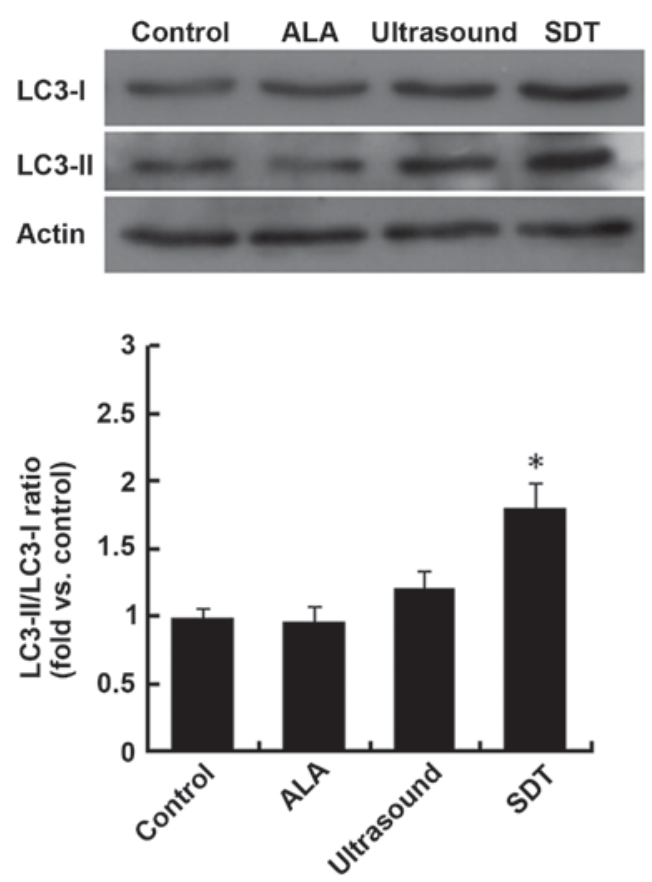

Figure 5. PpXT-SDT increases the ratio of LC3-II/LC3-I in VSMCs. The ratios of LC3-II/LC3-I were quantified by Quantity One software. Data are presented as mean $\pm \mathrm{SD}$ of three independent assessments. ${ }^{*} \mathrm{P}<0.05$ vs. control. in the SDT group compared to the control group. Thus, we conclude that SDT promotes VSMC autophagic activity.

\section{Discussion}

Recently, Michiels et al, reported that PpIX-SDT promoted VSMC phenotype transformation from a dedifferentiated to a differentiated status (14), which partially explained why SDT inhibited in-stent restenosis in animal models (4). PpIX has low toxicity and a short dark period in the cells, as compared with other sonosensitizers $(15,16)$. In addition, Michiels et al (14) showed that PpIX-SDT had no effect on VSMC viability. Consistent with the above findings, we did not observe that PpIX-SDT altered the viability of VSMCs, despite having increased the ultrasound intensity to $1.0 \mathrm{~W} / \mathrm{cm}^{2}$. Together, these findings suggest that PpIX-SDT is a relatively safe therapeutic approach for treating atherosclerotic plaques.

Previously, Cheng et al $(17,18)$ reported that PpIX-SDT induced cell death in THP-1 macrophages via a mitochondria-dependent pathway. However, our results showed that PpIX-SDT did not significantly induce cell apoptosis compared to the control group in VSMCs, which coincided with the finding that MMP was not altered by PpIX-SDT treatment. The maintenance of MMP observed in our study is probably 
attributed to the limited ultrasonic energy and acoustic sensitivity agent concentration.

Cell autophagy is generally regarded as a cell survival mechanism because cells degrade non-essential, aging or non-functional proteins, and/or cytoplasmic organelles, and recycle those degraded components to maintain normal cellular homeostasis (19). Increasing evidence suggests that autophagy is involved in a wide range of diseases, including atherosclerosis $(20,21)$. During the development of atherosclerotic plaques, harmful material, such as a large number of reactive oxygen species, cause oxidative stress, leading to the maintenance of basal activity of cell autophagy, which can protect cells from the oxidative stress and promote plaque stability (22-24). Therefore, these cells were protected from cell death, i.e. apoptosis, and atherosclerotic plaques can form and steadily grow. Platelet derived growth factor (PDGF) was shown to mediate autophagy and adjust the response of VSMCs to phenotypic transformation induced by oxidative damage (25). However, excessive activation of cell autophagy eventually resulted in plaque cell death, and tended to be harmful. Because of the elastic strength of the plaque's fibrous cap, which mostly depends on smooth muscle cells and their secreted collagen (26), VSMC death usually leads to unstable plaque (27), and even plaque rupture. Hence, VSMC survival plays an important role in plaque stability (26). During the formation of atherosclerotic plaques, cell autophagy induced by mild oxidative stress contributes to the removal of damaged organelles. However, if induced autophagic activity is not sufficient to eliminate all damaged cell components, excessive oxidative stress and mitochondrial cytochrome $\mathrm{C}$ release can induce cell apoptosis. Therefore, it is generally believed that low levels of VSMC autophagy promote plaque stability, but high levels of cell autophagy are not conducive to stable plaques $(9,28)$.

In our study, we observed increased autophagic activity following SDT treatment, which was supported by the following evidence: i) increased autophagosome formation revealed by electron microscopy, ii) increased protein levels of LC3B, an autophagy molecular marker, revealed by immunofluorescence staining, and iii) an increased LC3-II/LC3-I ratio, as revealed by western blot analysis. LC3, originally named microtubule associated protein $1 \mathrm{~A}$ and $1 \mathrm{~B}$, referred to as MAP1LC3, plays an important role in autophagy. The mammalian LC3 family has three members: LC3A, LC3B and LC3C. Once synthesized, the C-terminus of LC3 is immediately sheared by autophagy related protein 4 (Atg4) and subsequently produces LC3-I, which is localized in the cytoplasm. In autophagy, LC3-I will be modified by Atg7 and Atg3 and subsequently generate LC3-II, which is located in the autophagosome. Thus, LC3 is widely recognized as an autophagy molecular marker (29), and the LC3-II/LC-I ratio may be used to evaluate the activity of autophagic flux in cells/organs. Taken together, the findings suggest that VSMC autophagy increases following PpIX-SDT treatment.

Autophagy is crucial for normal VSMC function, phenotype, and survival. Inhibition of autophagy as a therapeutic strategy in the treatment of neointimal stenosis and atherosclerosis would be unfavorable. Conversely, stimulation of autophagy could be a valuable new strategy in the treatment of arterial disease (30-33). Our study demonstrates that PpIX-SDT induces autophagy in cultured VSMCs. Future studies are required to elucidate the molecular mechanisms by which PpIX-SDT activates autophagy, and to explore whether SDT promotes plaque stability in in vivo atherosclerotic models.

In the present study, we demonstrate that PpIX-SDT induces VSMC autophagy, characterized by increased autophagasome formation. In addition, we show that PpIX-SDT does not trigger any significant increase in apoptosis or decrease in MMP. These results point to the possibility that induction of VSMC autophagy may be one of the mechanisms whereby SDT promotes plaque stability in vivo.

\section{Acknowledgements}

This study was supported by the National Natural Science Foundation of China (8140027).

\section{References}

1. Perelman A, Wachtel C, Cohen M, Haupt S, Shapiro H and Tzur A: JC-1: Alternative excitation wavelengths facilitate mitochondrial membrane potential cytometry. Cell Death Dis 3: e430, 2012.

2. Chen H, Gao W, Yang Y, Guo S, Wang H, Wang W, Zhang S, Zhou Q, Xu H, Yao J, et al: Inhibition of VDAC1 prevents $\mathrm{Ca}^{2+}$-mediated oxidative stress and apoptosis induced by 5-aminolevulinic acid mediated sonodynamic therapy in THP-1 macrophages. Apoptosis 19: 1712-1726, 2014.

3. Guo S, Sun X, Cheng J, Xu H, Dan J, Shen J, Zhou Q, Zhang Y, Meng L, Cao W and Tian Y: Apoptosis of THP-1 macrophages induced by protoporphyrin IX-mediated sonodynamic therapy. Int J Nanomedicine 8: 2239-2246, 2013.

4. Li X, Gao L, Zheng L, Kou J, Zhu X, Jiang Y, Zhong Z, Dan J, $\mathrm{Xu} \mathrm{H}$, Yang Y, et al: The efficacy and mechanism of apoptosis induction by hypericin-mediated sonodynamic therapy in THP-1 macrophages. Int J nanomedicine 10: 821-838, 2015.

5. Li Z, Sun X, Guo S, Wang L, Wang T, Peng C, Wang W, Tian Z, Zhao R, Cao W and Tian Y: Rapid stabilisation of atherosclerotic plaque with 5-aminolevulinic acid-mediated sonodynamic therapy. Thromb Haemost 4: 793-803, 2015.

6. Sun X, Xu H, Shen J, Guo S, Shi S, Dan J, Tian F, Tian Y and Tian Y: Real-time detection of intracellular reactive oxygen species and mitochondrial membrane potential in THP-1 macrophages during ultrasonic irradiation for optimal sonodynamic therapy. Ultrason Sonochem 22: 7-14, 2015.

7. Krammer B and Plaetzer K: ALA and its clinical impact, from bench to beside. Photochem Photobiol Sci 7: 283-289, 2008.

8. Peng C, Li Y, Liang H, Cheng J, Li Q, Sun X, Li Z, Wang F, Guo Y, Tian Z, et al: Detection and photodynamic therapy of inflamed atherosclerotic plaques in the carotid artery of rabbits. J Photochem Photobiol B 102: 26-31, 2011.

9. Mizushima N, Yoshimori T and Levine B: Methods in mammalian autophagy research. Cell 140: 313-326, 2010.

10. Martinet W and De Meyer GR: Autophagy in atherosclerosis. Curr Atheroscler Rep 10: 216-223, 2008.

11. Lavandero S, Chiong M, Rothermel BA and Hill JA: Autophagy in cardiovascular biology. J Clin Invest 125: 55-64, 2015.

12. Ouimet M, Franklin V, Mak E, Liao X, Tabas I and Marcel YL: Autophagy regulates cholesterol efflux from macrophage foam cells via lysosomal acid lipase. Cell Metab 13: 655-667, 2011.

13. Li BH, Yin YW, Liu Y, Pi Y, Guo L, Cao XJ, Gao CY, Zhang LL and Li JC: TRPV1 activation impedes foam cell formation by inducing autophagy in oxLDL-treated vascular smooth muscle cells. Cell Death Dis 5: e1182, 2014.

14. Michiels CF, Fransen P, De Munck DG, De Meyer GR and Martinet W: Defective autophagy in vascular smooth muscle cells alters contractility and $\mathrm{Ca}^{2+}$ homeostasis in mice. Am J Physiol Heart Circ Physiol 308: H557-H567, 2015.

15. Dan J, Sun X, Li W, Zhang Y, Li X, Xu H, Li Z, Tian Z, Guo S, Yao J, et al: 5-Aminolevulinic acid-mediated sonodynamic therapy promotes phenotypic switching from dedifferentiated to differentiated phenotype via reactive oxygen species and p38 mitogen-activated protein kinase in vascular smooth muscle cells. Ultrasound Med Biol 41: 1681-1689, 2015. 
16. Hagiya Y, Fukuhara H, Matsumoto K, Endo Y, Nakajima M, Tanaka T, Okura I, Kurabayashi A, Furihata M, Inoue K, et al: Expression levels of PEPT1 and ABCG2 play key roles in 5-aminolevulinic acid (ALA)-induced tumor-specific protoporphyrin IX (PpIX) accumulation in bladder cancer. Photodiagnosis Photodyn Ther 10: 288-295, 2013.

17. Uto Y, Tamatani D, Mizuki Y, Endo Y, Nakanishi I, Ohkubo K, Fukuzumi S, Ishizuka M, Tanaka T, Kuchiike D, et al: Evaluation of the sonosensitizing activities of 5-aminolevulinic acid and $\mathrm{Sn}(\mathrm{IV})$ chlorin e6 in tumor-bearing chick embryos. Anticancer Res 34: 4583-4587, 2014.

18. Cheng J, Sun X, Guo S, Cao W, Chen H, Jin Y, Li B, Li Q, Wang H, Wang Z, et al: Effects of 5-aminolevulinic acid-mediated sonodynamic therapy on macrophages. Int J Nanomedicine 8: 669-676, 2013.

19. Li Y, Zhou Q, Hu Z, Yang B, Li Q, Wang J, Zheng J and Cao W: 5-Aminolevulinic acid-based sonodynamic therapy induces the apoptosis of osteosarcoma in mice. PLoS One 10: e0132074, 2015.

20. Kundu M and Thompson CB: Autophagy: Basic principles and relevance to disease. Annu Rev Pathol 3: 427-455, 2008.

21. Luo Y, Lu S, Zhou P, Ai QD, Sun GB and Sun XB: Autophagy: An exposing therapeutic target in atherosclerosis. J Cardiovasc Pharmacol 67: 266-274, 2016.

22. Perrotta I and Aquila S: The role of oxidative stress and autophagy in atherosclerosis. Oxid Med Cell Longev 2015: 130315, 2015.

23. Liao X, Sluimer JC, Wang Y, Subramanian M, Brown K, Pattison JS, Robbins J, Martinez J and Tabas I: Macrophage autophagy plays a protective role in advanced atherosclerosis. Cell Metab 15: 545-553, 2012.

24. Mehta JL, Chen J, Hermonat PL, Romeo F and Novelli G: Lectin-like, oxidized low-density lipoprotein receptor-1 (Lox-1): A critical player in the development of atherosclerosis and related disorders. Cardiovasc Res 69: 36-45, 2006.

25. Xu K, Yang Y, Yan M, Zhan J, Fu X and Zheng X: Autophagy plays a protective role in free cholesterol overload-induced death of smooth muscle cells. J Lipid Res 51: 2581-2590, 2010.
26. Salabei JK, Cummins TD, Singh M, Jones SP, Bhatnagar A and Hill BG: PDGF-mediated autophagy regulates vascular smooth muscle cell phenotype and resistance to oxidative stress. Biochem J 451: 375-388, 2013.

27. Mahmoudi MJ, Mahmoudi M, Siassi F, Shokri F, Eshraghian MR, Zarnani AH, Chahardoli R, Hedayat M, Khoshnoodi J, Nayeri H, et al: Lymphocyte cytotoxicity of oxLDL in patients with atherosclerosis. Iran J Immunol 8: 27-33, 2011.

28. Mehta JL, Chen J, Hermonat PL, Romeo F and Novelli G: Lectin-like, oxidized low-density lipoprotein receptor-1 (LOX-1): A critical player in the development of atherosclerosis and related disorders. Cardiovasc Res 69: 36-45, 2006.

29. Martinet W and De Meyer GR: Autophagy in atherosclerosis: A cell survival and death phenomenon with therapeutic potential. Circ Res 104: 304-317, 2009.

30. Kabeya Y, Mizushima N, Ueno T, Yamamoto A, Kirisako T, Noda T, Kominami E, Ohsumi Y and Yoshimori T: LC3, a mammalian homogue of yeast Apg8p, is localizing in autophagosome membranes after processing. EMBO J 19: 5720-5728, 2000.

31. Grootaert MO, da Costa Martins PA, Bitsch N, Pintelon I, De Meyer GR, Martinet W and Schrijvers DM: Defective autophagy in vascular smooth muscle cells accelerates senescence and promotes neointima formation and atherogenesis. Autophagy 11: 2014-2032, 2015.

32. Klionsky DJ, Abeliovich H, Agostinis P, Agrawal DK, Aliev G, Askew DS, Baba M, Baehrecke EH, Bahr BA, Ballabio A, et al: Guidelines for the use and interpretation of assays for monitoring autophagy in higher eukaryotes. Autophagy 4: 151-175, 2008.

33. Salabei JK, Cummins TD, Singh M, Jones SP, Bhatnagar A and Hill BG: PDGF-mediated autophagy regulates vascular smooth muscle cell phenotype and resistance to oxidative stress. Biochem J 451: 375-388, 2013 